\title{
Local adaptation, geographical distance and phylogenetic relatedness: Assessing the drivers of siderophore-mediated social interactions in natural bacterial communities
}

\author{
Elena Butaitè $^{1}$ (D) | Jos Kramer ${ }^{1,2}$ (D) | Rolf Kümmerli, ${ }^{1,2}$ (D)
}

${ }^{1}$ Department of Plant and Microbial Biology, University of Zurich, Zurich, Switzerland

${ }^{2}$ Department of Quantitative Biomedicine, University of Zurich, Zurich, Switzerland

\section{Correspondence}

Rolf Kümmerli, Department of Quantitative Biomedicine, University of Zurich, Winterthurerstrasse 190, 8057 Zurich, Switzerland.

Email: rolf.kuemmerli@uzh.ch

\section{Funding information}

Schweizerischer Nationalfonds zur Förderung der Wissenschaftlichen Forschung, Grant/Award Number: 165835 and 182499; Deutsche Forschungsgemeinschaft, Grant/Award Number: KR 5017/2-1; H2020 European Research Council, Grant/Award Number: 681295; Forschungskredit der Universität Zürich, Grant/Award Number: FK-15-082

\begin{abstract}
In heterogenous, spatially structured habitats, individuals within populations can become adapted to the prevailing conditions in their local environment. Such local adaptation has been reported for animals and plants, and for pathogens adapting to hosts. There is increasing interest in applying the concept of local adaptation to microbial populations, especially in the context of microbe-microbe interactions. Here, we tested whether cooperation and cheating on cooperation can spur patterns of local adaptation in soil and pond communities of Pseudomonas bacteria, collected across a geographical scale of 0.5 to $50 \mathrm{~m}$. We focussed on the production of pyoverdines, a group of secreted iron-scavenging siderophores that often differ among pseudomonads in their chemical structure and the receptor required for their uptake. A combination of supernatant-feeding and competition assays between isolates from four distance categories revealed tremendous variation in the extent to which pyoverdine non- and low-producers can benefit from pyoverdines secreted by producers. However, this variation was not explained by geographical distance, but primarily depended on the phylogenetic relatedness between interacting isolates. A notable exception occurred in local pond communities, where the effect of phylogenetic relatedness was eroded in supernatant assays, probably due to the horizontal transfer of receptor genes. While the latter result could be a signature of local adaptation, our results overall indicate that common ancestry and not geographical distance is the main predictor of siderophore-mediated social interactions among pseudomonads.
\end{abstract}

\section{KEYWORDS}

genetic isolation-by-distance, microbe-microbe interactions, Pseudomonas, public goods, siderophores

\section{1 | INTRODUCTION}

Local adaptation in spatially structured environments, where dispersal of individuals is limited and environmental patches differ in conditions, is a common phenomenon in animals and plants (Blanquart et al., 2013; Hereford, 2009; Kawecki \& Ebert, 2004; Leimu \& Fischer, 2008; Savolainen et al., 2013). The concept implies that divergent selection causes local populations to adapt to abiotic 
and biotic conditions in their respective habitat patch. As a consequence, individuals cope well with prevailing local conditions, but potentially perform poorly (i.e. are mal-adapted) in other patches where conditions differ.

In contrast to animals and plants, the extent to which local adaptation occurs in microbial populations is much less well understood (Giraud et al., 2017; Kraemer \& Boynton, 2017). The majority of work in this field focussed on host-pathogen interactions and examined whether microbial pathogens (i.e. eukaryotic parasites, bacteria, viruses) are locally adapted to their hosts (Greischar \& Koskella, 2007; Kaltz \& Shykoff, 1998). Even fewer studies are available on local adaptation to abiotic conditions (Kraemer \& Boynton, 2017), with the existing body of work yielding mixed results. For example, Belotte et al. (2003) found significant patterns of local growth adaptation among a collection of Bacillus soil isolates across distances of up to $80 \mathrm{~m}$, while others could not confirm such patterns for Pseudomonas isolates sampled across a geographical scale of 10-1,000 m (Kraemer \& Kassen, 2015, 2016).

In addition to abiotic conditions, there is an increasing interest in understanding whether bacteria can locally adapt to other members of their community (Kraemer \& Boynton, 2017). The reason is that bacteria possess numerous traits to socially interact with other microbes (West et al., 2007). Social interactions can include both cooperative interactions, for example through the sharing of secreted enzymes or nutrient scavenging molecules (Abisado et al., 2018; Asfahl \& Schuster, 2017; Kramer, Özkaya et al., 2020) and competitive interactions, for example, through the secretion of toxins (Abrudan et al., 2015; Ghoul \& Mitri, 2016; Hibbing et al., 2010). Microbial toxins such as bacteriocins and antibiotics exert narrowrange activities against closely related strains with considerable niche overlap. Their deployment could hence spur patterns of local adaptation so that bacteria are particularly efficient in combatting their local competitors (Bruce et al., 2017; Hawlena et al., 2010; Kinkel et al., 2014). However, results on local adaptation with regard to toxin-mediated social interactions are scarce and mixed. While some studies revealed significant geographical distance effects in Xenorhabdus and Streptomyces populations collected from soil (Hawlena et al., 2010; Kinkel et al., 2014), with inhibition being most pronounced against strains from distant patches, other studies found no or very weak evidence for local adaptation in Pseudomonas soil populations (Bruce, West et al., 2017; Kraemer et al., 2017). The latter studies further found that strain inhibition via bacteriocins was low overall $(<10 \%)$. Bacteriocins and their immunity proteins are often encoded on mobile genetic elements (Brockhurst et al., 2019; Nogueira et al., 2009; Silby et al., 2011) and can thus spread quickly as selfish genetic elements through populations, which might explain both the low frequency of inhibition and the absence of local adaptation.

In our study, we examine whether cooperative interactions and cheating on a cooperative trait can trigger patterns of local adaptation in bacterial communities. We focus on pyoverdine, an iron-chelating siderophore produced by Pseudomonas bacteria. Siderophore production can be a cooperative trait because the molecules are secreted in the environment where they scavenge iron from natural sources, making it bioavailable for community members in the vicinity that possess a matching receptor for the uptake of the chelated iron (Kramer, Özkaya et al., 2020). There is increasing evidence that the sharing of siderophores and their exploitation by siderophore nonproducers drive community dynamics in marine, fresh-water and soil environments (Bruce et al., 2017; Butaite et al., 2017; Cordero et al., 2012; Gu et al., 2020; Kramer, Özkaya et al., 2020). Given their strong fitness effects, local adaptation to siderophore use and exploitation could manifest in two different ways. First, siderophore nonproducers could become locally adapted to efficiently exploit siderophores produced by members of their own community. Second, producers could evolve strategies to minimize exploitation by local nonproducers.

At the mechanistic level, such local adaptation could arise due to variation in the chemical structure of siderophores and their corresponding receptors required for uptake (Faraldo-Gómez \& Sansom, 2003; Hider \& Kong, 2010; Kümmerli et al., 2014). In this respect, pyoverdine, the main siderophore produced by fluorescent pseudomonads, is an ideal model trait, because many different varieties of this molecule and its receptor exist (Butaitè et al., 2017; Ghysels et al., 2004; Meyer et al., 2008; Smith et al., 2005). While pyoverdines feature a conserved fluorophore, their peptide backbone can vary substantially and is usually strain-specific (Meyer et al., 2008; Schalk \& Guillon, 2013; Visca et al., 2007). It was suggested that the observed diversity could be the result of antagonistic co-evolution, whereby pyoverdine nonproducers that experience a relative fitness advantage by exploiting pyoverdine produced by others, that is, cheaters, trigger selection for novel, less-exploitable variants of pyoverdines. This process could in turn lead to selection for novel, more efficient cheater types (Butaitè et al., 2017; Lee et al., 2012; Smith et al., 2005). In structured populations with limited dispersal, such antagonistic co-evolution could occur within patches and then give rise to cheaters that are proficient in exploiting local producers, but might be less efficient in exploiting producers from more distant patches with which they have not interacted before.

To explore this scenario, we isolated a total of 315 Pseudomonas strains from eight soil and eight pond samples (henceforth called 'communities', each comprising 18-20 isolates) across a geographical scale of $50 \mathrm{~m}$ for each habitat (Figure 1a). We identified pyoverdine nonproducers and low-producers (i.e. potential cheaters, henceforth called NLPs) and tested in a common-garden experiment whether there are differences in the extent to which these NLPs can use the pyoverdine from producers originating either from their own community or different communities at close $(0.5 \mathrm{~m})$, intermediate $(5 \mathrm{~m})$ and far $(50 \mathrm{~m})$ distances. We used two complementary assays. First, we measured the growth effects NLPs experience when being exposed to pyoverdine in supernatants from producers. Second, we measured the relative fitness of NLPs in direct competition with the producers. In direct competition, NLPs and producers might induce additional competitive traits (e.g. contact-dependent killing, toxins) that influence fitness but are not captured by the supernatant assay. It is thus important to verify whether pyoverdine-mediated growth 

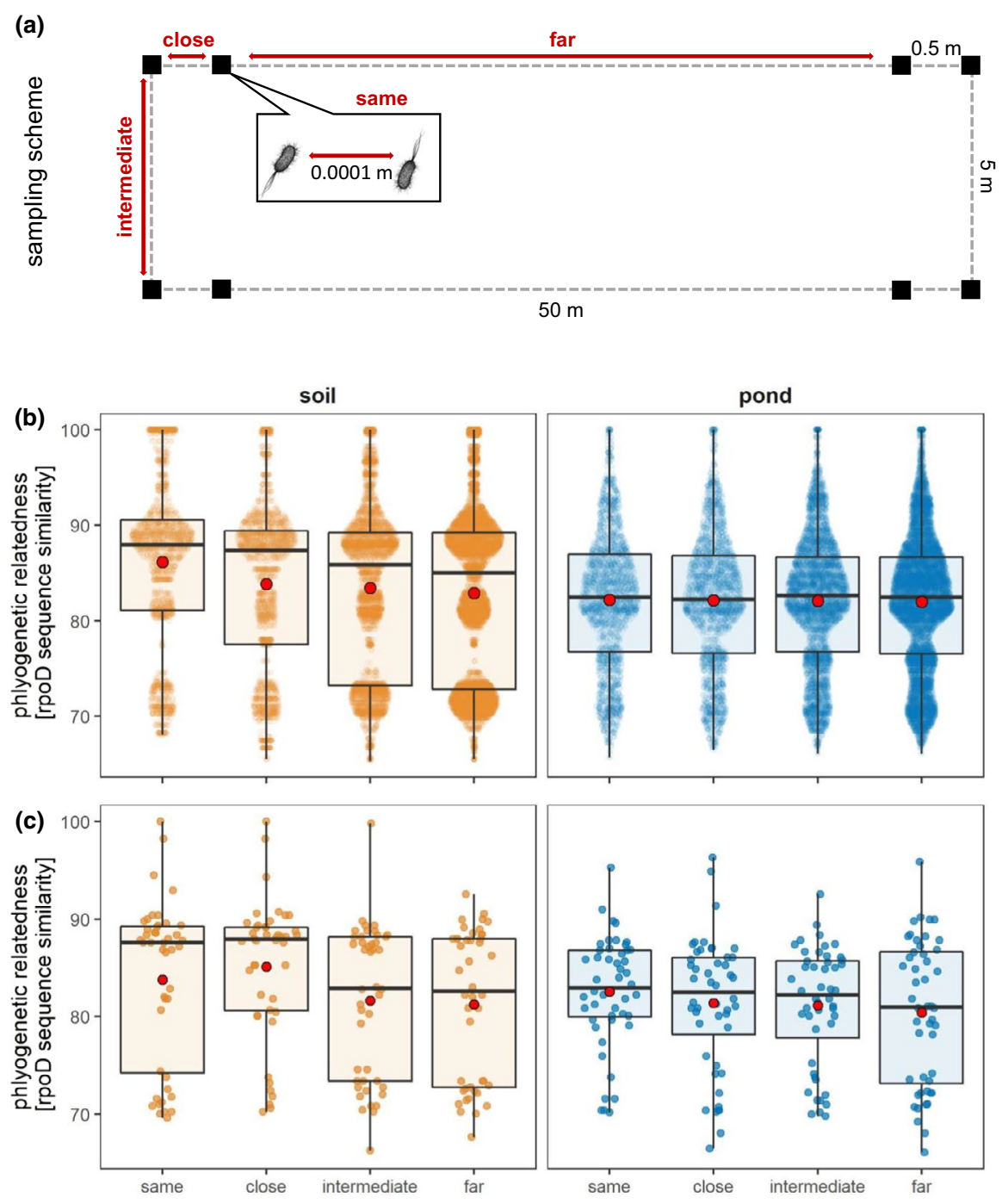

FIGURE 1 Sampling design and phylogenetic relatedness by distance. (a) Scheme of the sampling design used for both the soil and the pond habitat. Black squares represent the eight independent soil and water communities sampled, and the distances between them. (b) Phylogenetic relatedness between the full collection of 154 soil (yellow) and 150 pond (blue) isolates across the four different distance categories, based on the total of 45,912 (within-habitat) pairwise comparisons of partial rpoD sequences. Phylogenetic relatedness significantly declines over distance in the soil but not in the pond. Data were analysed with a Mantel correlation test for which the distance between isolates from the same community had to be set to a small value larger than zero (i.e. $0.0001 \mathrm{~m})$. (c) Phylogenetic relatedness between the isolates used in the supernatant assay: pyoverdine producers (soil: 88, pond: 87) versus pyoverdine NLPs (soil: 11, pond: 12), resulting in a total of 368 isolate combinations (soil: 176, pond: 192) effects translate into relative fitness consequences in direct competition. Finally, we used sequence data from the rpoD gene to test whether there is genetic isolation-by-distance between interacting isolates across the geographical scale sampled, and whether patterns of pyoverdine-mediated interactions correlate with the genetic relatedness between the interacting isolates.

\section{2 | MATERIALS AND METHODS}

\section{1 | Sampling and isolation of pseudomonads}

We sampled eight soil cores and eight water samples from a meadow and a pond on the Irchel campus of the University of Zurich $\left(47.40^{\circ} \mathrm{N}\right.$, $8.54^{\circ} \mathrm{E}$ ), Switzerland. We followed a rectangular collection scheme (Figure 1a), such that each soil core and water sample had one close (50 cm apart), two intermediate $(5 \mathrm{~m})$ and four distant $(50 \mathrm{~m})$ neighbouring samples. The isolation and characterization of fluorescent Pseudomonas spp. from these samples are described in detail in two previous papers (Butaitè et al., 2017, 2018). In brief, bacterial suspensions from soil and pond extracts were plated on Gould's S1 medium supplemented with $100 \mu \mathrm{g} / \mathrm{ml}$ of the antifungal cycloheximide and $50 \mu \mathrm{M} \mathrm{FeCl}_{3}$ (to also allow siderophore nonproducers to grow). This medium is selective for fluorescent Pseudomonas (Gould et al., 1985). Following three days of incubation at room temperature, we randomly picked 20 isolates for each of the eight soil and eight pond samples, 320 isolates in total, re-streaked them on lysogeny broth (LB) agar, and finally picked a single colony for freezer stock preservation at $-80^{\circ} \mathrm{C}$ in a $25 \%$-glycerol-LB mix. Each isolate was provided with an ID code, and isolates from the same sample were considered to belong to the same bacterial community.

\subsection{Genetic characterization of isolates and phylogenetic analysis}

We have previously sequenced the housekeeping gene rpoD of all 320 isolates (see Butaite et al., 2017), for detailed protocols and primers). PCR amplification and sequencing were successful for 315 isolates, while it failed in the remaining five strains, which 
consequently had to be excluded from further analysis. The rpoD gene is commonly used for phylogenetic affiliation of pseudomonads (Ghyselinck et al., 2013; Mulet et al., 2009). We performed in-depth phylogenetic analysis elsewhere (Butaitè et al., 2017; Kramer, Lopez Carrasco et al., 2020), confirming that our 315 isolates belong to the group of fluorescent pseudomonads. For this paper, we estimated the phylogenetic relatedness between interacting strains by carrying out a multiple sequence alignment and obtaining a pairwise identity matrix for $513 \mathrm{bp} \mathrm{rpoD}$ sequences of 304 strains, using MAFFT (Madeira et al., 2019). Eleven isolates had to be excluded at this stage because their sequence lengths were too short, such that their inclusion would have compromised the resolution in calculating the relatedness.

\section{3 | Experimental pairing of isolates}

In our previous paper, we screened all isolates for their ability to produce pyoverdine and to grow under iron-limited conditions (Butaite et al., 2017). Specifically, we made use of the auto-fluorescent property of pyoverdine and measured its production level through excitation at $400 \mathrm{~nm}$ and emission at $460 \mathrm{~nm}$, in iron-limited medium. This assay revealed that 28 out of the 315 isolates produced no or less than $5 \%$ of the pyoverdine produced by characterized laboratory Pseudomonas reference strains (e.g. including P. aeruginosa PAO1, P. protegens CHAO, P. putida IsoF; see detailed description in Butaite et al., 2017). We considered these 28 isolates as pyoverdine nonproducers and thus potential cheaters. Furthermore, there was high variation in the pyoverdine levels among producers, with many isolates producing less than $50 \%$ of the pyoverdine produced by the reference strains. We considered this latter group of isolates as pyoverdine low-producers, which could be partial cheaters (Ghoul et al., 2014). We henceforth abbreviate non- and low-producers as NLPs.

For the current paper, we picked one or two NLPs (upon availability) from each community and used them as focal isolates to test whether they are locally adapted to exploit pyoverdine from producers. Overall, we had 14 nonproducers and 10 lowproducers, originating from seven soil communities (i.e. one soil community featured no NLPs) and eight pond communities. We paired each of the 24 focal isolates with four random producers from each of the four distance categories (i.e. same, close, intermediate, or distant community; 16 producers per focal strain). For producers to be included, they had to: (a) grow better than the corresponding NLPs under iron-limited conditions; (b) produce more pyoverdine than the NLPs they were combined with; and (c) differ in the $r p o D$ sequence from the other three producers of the same community, and thus represent phylogenetically different strains. These criteria were met for all but one focal isolate that had to be paired with three producers with identical rpoD sequences from the same community, as no other options were available. This design resulted in 384 NLP-producer combinations (192 per habitat type). Given the limited number of producers available, some of them were combined with multiple focal isolates, so that our design finally featured 179 different pyoverdine producers (92 and 87 isolates from soil and pond, respectively).

\subsection{Supernatant assay}

To quantify the extent to which NLPs can use pyoverdine from producers, we harvested pyoverdine-containing supernatants from all the producers and fed them to the focal NLPs. We followed the protocol described in our previous study (Butaite et al., 2018). Specifically, we grew all producers (from overnight LB cultures) in CAA medium (5 g casamino acids, $1.18 \mathrm{~g} \mathrm{~K}_{2} \mathrm{HPO}_{4} \cdot 3 \mathrm{H}_{2} \mathrm{O}, 0.25 \mathrm{~g}$ $\mathrm{MgSO}_{4} \cdot 7 \mathrm{H}_{2} \mathrm{O}$ per litre) supplemented with $200 \mu \mathrm{M}$ 2,2'-dipyridyl as the iron chelator to stimulate pyoverdine production. Producers were grown in a total volume of $2 \mathrm{ml}$ in 24 -well plates, static at $25^{\circ} \mathrm{C}$ for $18 \mathrm{hr}$. Subsequently, we centrifuged cultures for $10 \mathrm{~min}$ at 3,500 rpm (Eppendorf Centrifuge 5804R) and transferred $900 \mu \mathrm{l}$ of the supernatants to AcroPrep Advance 96-well $1 \mathrm{ml}$ filter plates (with a $0.2 \mu \mathrm{m}$ supor membrane; Pall Corporation), attached to an autoclaved $1.2 \mathrm{ml}$ 96-well PCR plate (VWR). We centrifuged the samples in the filter plates together with the collection plates for $15 \mathrm{~min}$ at 2,500 rpm. The collection plates with sterile supernatants were sealed with Greiner SILVERseals and stored at $-20^{\circ} \mathrm{C}$.

Next, we grew the NLPs in $200 \mu$ of LB in 96-well plates overnight static at $25^{\circ} \mathrm{C}$. Overnight, cultures of all focal strains were then adjusted to OD600 $=0.05$ (optical density at $600 \mathrm{~nm}$; measured with the microplate reader Infinite M200, Tecan Group Ltd.). $2 \mu \mathrm{l}$ of these adjusted cultures was transferred to four different variants of the CAA medium: (a) $180 \mu \mathrm{l}$ CAA supplemented with $200 \mu \mathrm{M} 2,2^{\prime}$ - dipyridyl and $20 \mu \mathrm{l}$ producer supernatant; (b) $180 \mu \mathrm{l}$ CAA supplemented with $200 \mu \mathrm{M}$ 2,2'- dipyridyl and $20 \mu \mathrm{l}$ CAA that underwent the same treatment as the supernatants including filtering and freezing; (c) $180 \mu \mathrm{l} \mathrm{CAA} \mathrm{supplemented} \mathrm{with} 40 \mu \mathrm{M} \mathrm{FeCl}_{3}$ and $20 \mu \mathrm{l}$ producer supernatant; (d) $180 \mu \mathrm{l} \mathrm{CAA} \mathrm{supplemented} \mathrm{with}$ $40 \mu \mathrm{M} \mathrm{FeCl}_{3}$ and $20 \mu \mathrm{l} \mathrm{CAA}$ that underwent the same treatment as the supernatants including filtering and freezing. While (a) is our main treatment to quantify the effect of supernatants on focal strain growth, relative to the growth in nonsupplemented medium (b), treatments (c) and (d) serve as controls to assess the effect of supernatants on growth under iron-rich conditions where pyoverdine is not important for iron acquisition.

Each treatment was repeated four times for each isolate combination. The plates were incubated statically for $15 \mathrm{hr}$ at $25^{\circ} \mathrm{C}$. The final OD600 of cultures was measured using the microplate reader. We then used the OD600 measurements to calculate the 'supernatant effect' under iron-limited conditions as [OD600 from treatment (a)]/[OD600 from treatment (b)], and under iron-rich conditions as [OD600 from treatment (c)]/[OD600 from treatment (d)]. All ratios were log-transformed and values larger or smaller than zero indicate growth stimulation or inhibition, respectively. One focal strain did not grow under iron-limited conditions, and the supernatant effect thus remained undefined. This strain had to be excluded from all subsequent analyses. 


\section{5 | Competition assays}

Next, we directly competed NLPs against pyoverdine producers under iron-limited conditions to test whether the pyoverdinemediated growth effects (as measured by the supernatant assay) translate into relative fitness consequences for the interacting strains. In order to distinguish the two competing strains, we integrated a single copy of a constitutively expressed mCherry marker into the chromosome of NLPs using the mini-Tn7 system (Choi \& Schweizer, 2006). We used both the electroporation and conjugation protocol by Choi and Schweizer (2006) with modifications described in Butaitè et al. (2017) for the successful tagging of 12 NLPs (six soil and six pond isolates), originating from five soil and five pond communities. Each of the 12 tagged isolates was competed against the corresponding 16 pyoverdine producers (four from each distance category) used for the supernatant assay. This resulted in 192 NLP-producer combinations (96 per habitat type).

The competition experiments entailed the following steps: (a) we grew isolates as monocultures overnight in $200 \mu \mathrm{l}$ LB medium in 96-well plates for about $17 \mathrm{hr}$ at $25^{\circ} \mathrm{C}$. (b) We adjusted the cultures to $\mathrm{OD} 600=0.05$ in LB. (c) We mixed strains in a 1:5 volumetric ratio $(20 \mu \mathrm{l}$ of mCherry-tagged NLPs culture with $100 \mu \mathrm{l}$ of the untagged producer culture). (d) We transferred $2 \mu \mathrm{l}$ of strain mixes, but also monocultures and media blanks as controls, to $200 \mu \mathrm{l}$ CAA containing $200 \mu \mathrm{M}$ 2,2'-dipyridyl in 96-well plates, in five-fold replication. (e) We let strains compete for about $46 \mathrm{hr}$ at $25^{\circ} \mathrm{C}$ under static conditions. (f) We used flow cytometry to estimate the initial and final frequencies of the competing strains (see detailed method below). Important to note is that we used filtered medium (passed through a $0.22 \mu \mathrm{m}$ filter) for all competition experiments to reduce the medium background signal during flow cytometry measurements. (g) We estimated the relative fitness of the NLPs as: $v=\left[a_{t}\left(1-a_{0}\right)\right] /\left[a_{0}\left(1-a_{t}\right)\right]$, where $a_{0}$ and $a_{t}$ are initial and the final frequencies of NLPs in the mixed cultures with the producer, respectively (Ross-Gillespie et al., 2007). We log-transformed $v$ values, whereby $v<0$ indicates a decrease and $v>0$ an increase in the relative fitness of NLPs compared to its pyoverdine-producing competitor.

\section{6 | Flow cytometry}

Prior to flow cytometry measurements, we fixed cells (to stop any biological process) using paraformaldehyde. We first dissolved paraformaldehyde in PBS (phosphate-buffered saline) to obtain a $10 \%$ stock solution, and then further diluted the stock with $0.85 \% \mathrm{NaCl}$ to obtain a final concentration of $2 \%$. This solution was filter-sterilized using a $0.22 \mu \mathrm{m}$ filter. We used the paraformaldehyde-PBS- $\mathrm{NaCl}$ solution to fix and dilute both mono- and mixed cultures before (a representative aliquot) and after the competition period. Appropriate dilution factors were chosen to obtain less than 10,000 events/s, which is the upper limit our flow cytometer (LSR II Fortessa, BD
Biosciences) could handle. Cells were fixed for $20 \mathrm{~min}$ in the dark at room temperature and then kept at $4^{\circ} \mathrm{C}$ prior to staining. We stained fixed cells with Sybr Green I (Invitrogen; the commercial stock was diluted by $2 \times 10^{4} \mathrm{x}$ ) for $30 \mathrm{~min}$ at room temperature in the dark. Pre-competitions (mono- and mixed cultures) were analysed in triplicates, while competitions involved five independent replicates per strain combination. We used the HTS mode (automated 96-well sampler) of the flow cytometer to assess strain frequencies and total cell counts in $10 \mu \mathrm{l}$ per sample, at the flow cytometry core facility of the University of Zurich. Sybr Green I (excitation: 488 nm/emission: $530 \mathrm{~nm}$ ) and mCherry (excitation: $561 \mathrm{~nm} /$ emission: 610/20 nm) fluorescence was measured using the corresponding detection filters. We used the FlowJo software (Tree Star) for data gating and the calculations of strain frequencies. Because the mCherry signal was typically too weak in the pre-competition samples, we used Sybr Green I to assess cell counts in monocultures prior to mixing and used those to infer strain frequencies in the 1:5 volumetric mixes used to initiate the competitions. After the competition period, the $\mathrm{mCherry} \mathrm{signal} \mathrm{was} \mathrm{strong,} \mathrm{which} \mathrm{allowed} \mathrm{an} \mathrm{unambiguous} \mathrm{distinc-}$ tion between tagged and untagged cells.

\section{7 | Statistical analysis}

We first explored whether phylogenetic relatedness varied with geographical distance in our full collection of soil and pond isolates. To account for the possibility that phylogenetic diversity changes more distinctively over spatial scales in the more structured soil environment as compared to the more diffusive pond environment, we performed separate Mantel tests for soil and pond isolates. To further assess whether relatedness between NLP and producer pairs used in our experiments changes with distance, we fitted a linear mixed model (LMM) using phylogenetic relatedness as response variable, and habitat (soil or pond), distance category (same, close, intermediate or far), and their interaction as explanatory variables. To explore the potential determinants of the supernatant effect, we fitted a second LMM using the log-transformed supernatant effect as continuous response variable, and habitat, distance category, iron availability (iron-limited or iron-rich), phylogenetic relatedness (centred and scaled to unit variance) as well as all their interactions as explanatory variables. To finally assess the determinants of the outcome of direct competition between NLPs and producers, we fitted a third LMM using the (log-transformed) relative fitness values of the NLP as continuous response variable, and habitat, distance, relatedness, and their interactions as explanatory variables. Additionally, this third LMM contained the (log-transformed) supernatant effect and the initial ratio of the NLP as main effects to assess whether the supernatant effect was predictive of competitive outcomes, and whether these outcomes depended on variation in the relative starting frequency of the NLPs, respectively.

In our experiments, NLPs interacted with multiple pyoverdine producers and some pyoverdine producers were paired with 
multiple NLPs. To account for the resulting nonindependent repeated measurements, we initially fitted all models as random intercept models using NLP identity nested within their community, and producer identity nested within producer community as random effects. We then simplified models in a two-step procedure. First, we simplified the random component of the model based on likelihood ratio tests of model reduction. In a second step, we simplified the fixed component of each model by dropping nonsignificant interaction terms $(p>.05)$. All statistical analyses were conducted using the statistics software R version 3.5.0 (www.rproject.org). Mantel tests were performed using the 'mantel' function of the ecodist package with 9,999 permutations and 999 iterations for the bootstrapped confidence limits. Mixed models were implemented using the 'Imer' function of the Ime4 package. The $p$-values of effects in these models were obtained using the 'ANOVA' function of the car package and the 'summary' function of the ImerTest package.

\section{3 | RESULTS}

\subsection{Pseudomonas genetic relatedness varies across space in soil but not in pond}

In a first analysis, we compared the phylogenetic relatedness between pairs of isolates based on $r p o D$ sequence similarities across the four geographical distance categories of our sampling scheme (Figure 1a). When including all the 304 isolates with a sequence length $>500 \mathrm{bp}$, we found that phylogenetic relatedness decreased with geographical distance among soil isolates (Mantel R [upper $\mathrm{Cl}$; lower $\mathrm{Cl}]=-0.076$ [-0.108; -0.052$], p=.0001$; Figure $1 \mathrm{~b}$ ), but was independent of distance among pond isolates (Mantel R [upper $\mathrm{Cl}$; lower $\mathrm{Cl}]=-0.008$ [-0.017; -0.001$], p=.2019$; Figure $1 \mathrm{~b}$ ). When restricting the analysis to the isolate pairs we used for the supernatant assay, the phylogenetic relatedness did not differ between habitats and was overall independent of distance (LMM: habitat:
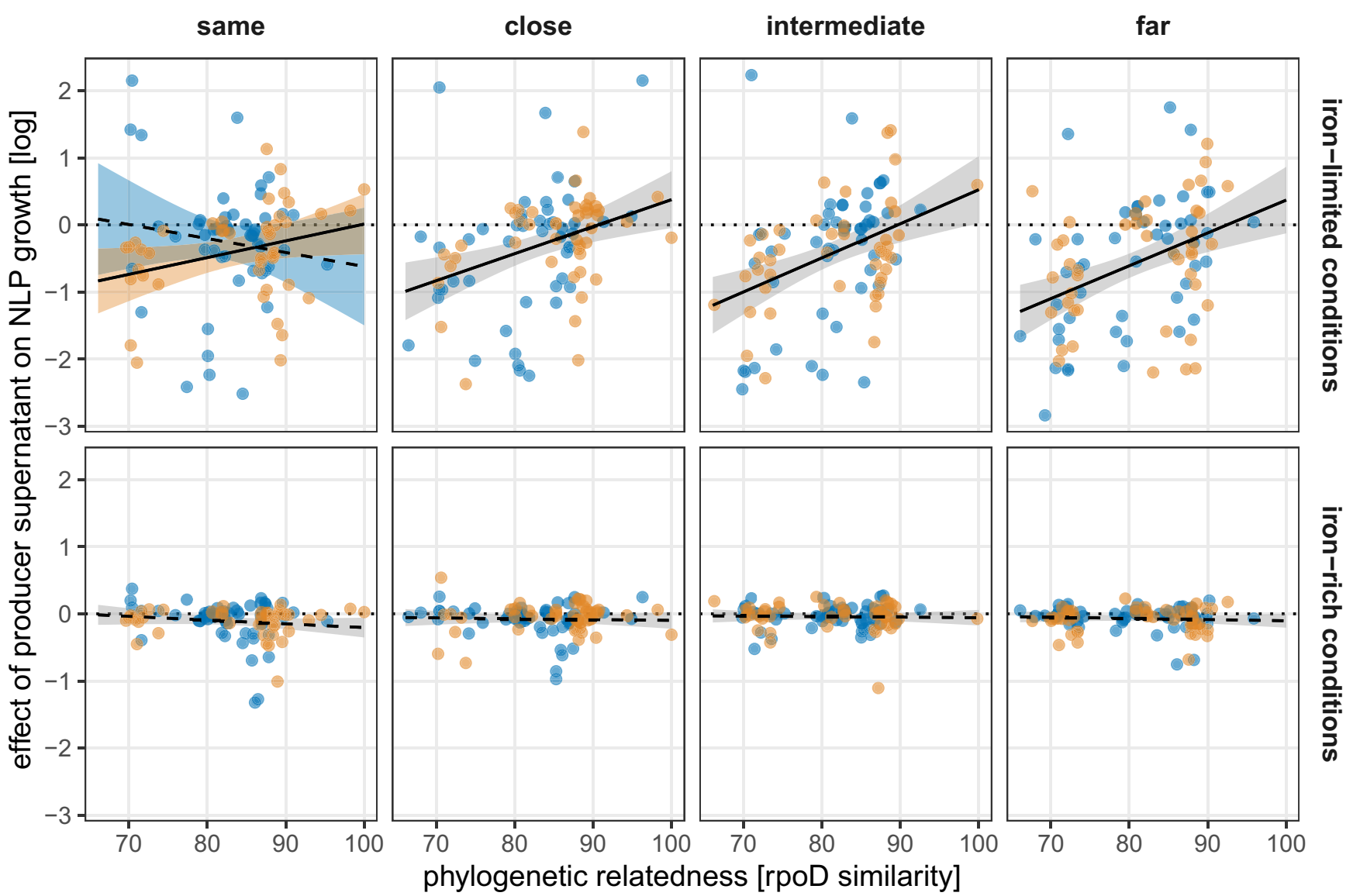

FIGURE 2 Under iron-limited conditions, the supernatant effect correlates positively with the phylogenetic relatedness between interacting isolates across all geographical distance categories, except for interactions among pond isolates from the same community. Depicted is the relationship between phylogenetic relatedness (based on $r p o D$ sequence similarities) between pairs of pyoverdine producers and NLPs (soil: 176, pond: 192) and the supernatant effect under iron-limited and iron-rich conditions for soil (yellow) and pond (blue) isolates across the four different geographical distance categories. The solid and dashed lines indicate significant and nonsignificant relationships, respectively. Dotted horizontal lines indicate the null line where supernatants have no effect on the growth of NLPs. Shaded areas are $95 \%$ confidence intervals; grey = relationship applying to both soil and pond isolates, yellow = soil-specific relationship, blue $=$ pond-specific relationship 
$\chi_{1}^{2}=1.472, p=.225$; distance: $\chi^{2}{ }_{3}=2.874, p=.412$; Figure 1c), a result that can likely be attributed to the reduced sample size and thus lower statistical power. Overall, our results show that there is weak but significant phylogenetic structuring of the Pseudomonas communities across a distance of $50 \mathrm{~m}$ in the soil, but not in the pond habitat.

\section{2 | Phylogenetic relatedness is the main predictor of the supernatant effect, yet geographical distance also plays a role among pond isolates}

To test whether pyoverdine-meditated social interactions vary across geographical distance, we fed pyoverdine-containing supernatants from 175 different producers to 23 focal pyoverdine non- and low-producers (NLPs), such that each NLP received supernatants from 16 producers originating from the four distance categories (Figure 1a; four producers per each distance category). Under iron-limited conditions where pyoverdine is important for growth, we observed 122 cases (soil: 56, pond: 66) in which the supernatant of producers stimulated the growth of NLPs (Figure 2). Because we know from our previous work that supernatant effects are predominantly driven by pyoverdine under iron limitation (Butaite et al., 2017), our results indicate that many NLPs can take up heterologous pyoverdines to overcome iron limitation. Conversely, we found 246 cases (soil: 120 , pond: 126 ) in which the supernatant of producers inhibited the growth of NLPs (Figure 2), suggesting that these isolates lack the ability to use the specific heterologous pyoverdine types fed.

When comparing these results across treatments and conditions, we found that the supernatant effect was shaped by an interaction between habitat, geographical distance and the relatedness between producer and NLP (Table S1). We therefore split the model and analysed the supernatant effect separately for soil and pond isolates. Among soil isolates, the supernatant effect was shaped by an interaction between iron limitation and phylogenetic relatedness (Table 1). Specifically, the supernatant effect increased with relatedness when iron was limited (slope $\pm \mathrm{SE}: 0.274 \pm 0.036, t_{337.1}=7.584$, $p<.001$; Figure 2), but was independent of relatedness under ironrich conditions $\left(0.022 \pm 0.036, t_{377.1}=0.619, p=.536\right.$; Figure 2). By contrast, the supernatant effect did not vary with geographical distance (Table 1; Figure 2). These findings suggest that there is no local adaptation with regard to pyoverdine-mediated social interactions (ranging from growth stimulation to inhibition) in Pseudomonas soil communities, but that interaction patterns are driven by phylogenetic relatedness between the isolates.

The patterns among pond isolates differed from those among soil isolates in one notable aspect (Figure 2): the supernatant effect depended on an interaction between iron limitation, phylogenetic relatedness and geographical distance (Table 1). This interaction arose because under iron-limited conditions, the supernatant effect was independent of phylogenetic relatedness among isolates from the same pond community $\left(-0.056 \pm 0.158, t_{187.4}=-0.354\right.$,
TABLE 1 Statistical analysis of the supernatant effect on NLPs. Influence of iron limitation, geographical distance and phylogenetic relatedness on the (log-transformed) effect of the supernatant of pyoverdine producers on the growth of pyoverdine non- and lowproducers (NLPs) from soil and pond communities

\begin{tabular}{|c|c|c|c|c|c|c|}
\hline & \multicolumn{6}{|c|}{ Supernatant effect [log] } \\
\hline & \multicolumn{3}{|l|}{ Soil } & \multicolumn{3}{|l|}{ Pond } \\
\hline & $\chi^{2}$ & $d f$ & $p^{*}$ & $\chi^{2}$ & $d f$ & $p^{*}$ \\
\hline Iron limitation (I) & 42.13 & 1 & $<.001$ & 28.45 & 1 & $<.001$ \\
\hline $\begin{array}{r}\text { Geographical } \\
\text { distance (D) }\end{array}$ & 2.07 & 3 & .558 & 0.87 & 3 & .833 \\
\hline $\begin{array}{l}\text { Phylogenetic } \\
\text { relatedness (R) }\end{array}$ & 29.14 & 1 & $<.001$ & 16.91 & 1 & $<.001$ \\
\hline I: D & $1.20^{\dagger}$ & $3^{\dagger}$ & $.753^{\dagger}$ & 2.87 & 3 & .413 \\
\hline $\mathrm{I}: \mathrm{R}$ & 28.69 & 1 & $<.001$ & 27.55 & 1 & $<.001$ \\
\hline$D: R$ & $0.24^{\dagger}$ & $3^{\dagger}$ & $.971^{\dagger}$ & 9.86 & 3 & .020 \\
\hline I: D: R & $1.31^{\dagger}$ & $3^{\dagger}$ & $.727^{\dagger}$ & 8.66 & 3 & .034 \\
\hline
\end{tabular}

*Significant $p$-values are given in bold.

${ }^{\dagger}$ Values before removal of interaction from the model.

$p=.724)$, but increased with phylogenetic relatedness among isolates from close $\left(0.469 \pm 0.125, t_{175.9}=3.761, p<.001\right)$, intermediate $\left(0.492 \pm 0.146, t_{184.1}=3.359, p<.001\right)$, and far communities $\left(0.509 \pm 0.123, t_{188.3}=4.154, p<.001\right.$; Figure 2$)$. These results show that the otherwise predominant effect of phylogenetic relatedness on the supernatant effect is eroded among members of the same pond community. Similar to the soil isolates, the supernatant effect was neither linked to phylogenetic relatedness $\left(\chi^{2}=0.15, d f=1\right.$, $p=.696)$ nor geographical distance $\left(\chi^{2}=6.80, d f=3, p=.075\right)$ under iron-rich conditions.

\subsection{The relative fitness of pyoverdine NLPs in direct competition with producers correlates with the results of the supernatant assay}

To examine whether the pyoverdine-mediated growth effects in the supernatant assay translate into relative fitness consequences in direct competitions between pyoverdine NLPs and producers, we competed the two types of isolates against each other using the same spatially arranged design, albeit with a reduced sample size. Overall, we had 192 producer-NLP combinations (soil: 96 , pond: 96 ), where each of the 12 NLPs was competed against 16 producers originating from the four distance categories (Figure 1a; four producers per each distance category).

We observed that competitions between pyoverdine producers and NLPs were mostly won by producers. In only 23 cases, we observed a relative fitness increase of the NLPs, whereas producers won in 169 cases. Overall, the relative fitness patterns were similar to those observed for the supernatant effects (Figure 3a). The relative fitness of NLPs increased with phylogenetic relatedness $\left(0.263 \pm 0.117, t_{155.0}=2.255, p=.024\right)$, but neither varied with 
(a)

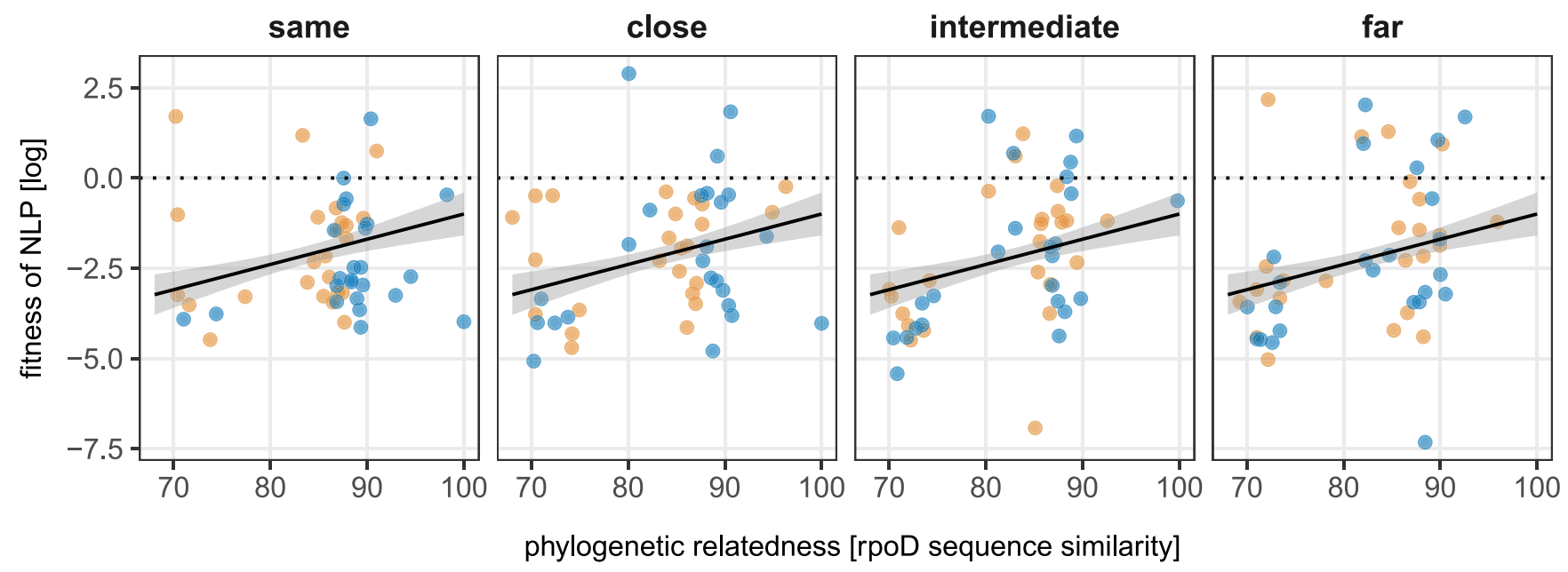

(b)

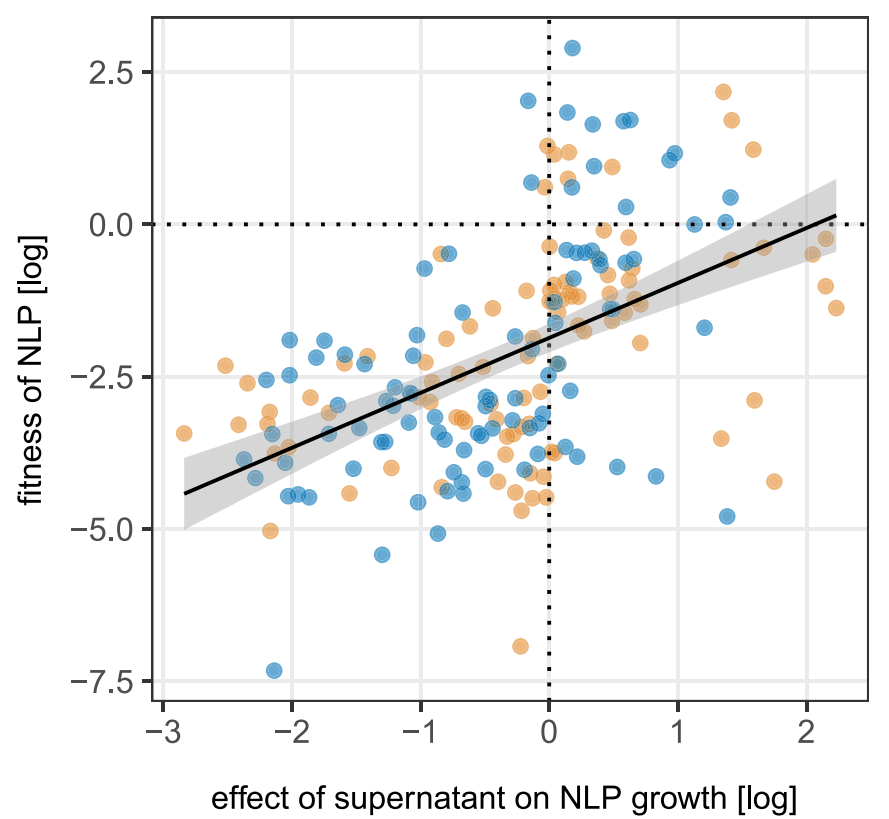

(c)

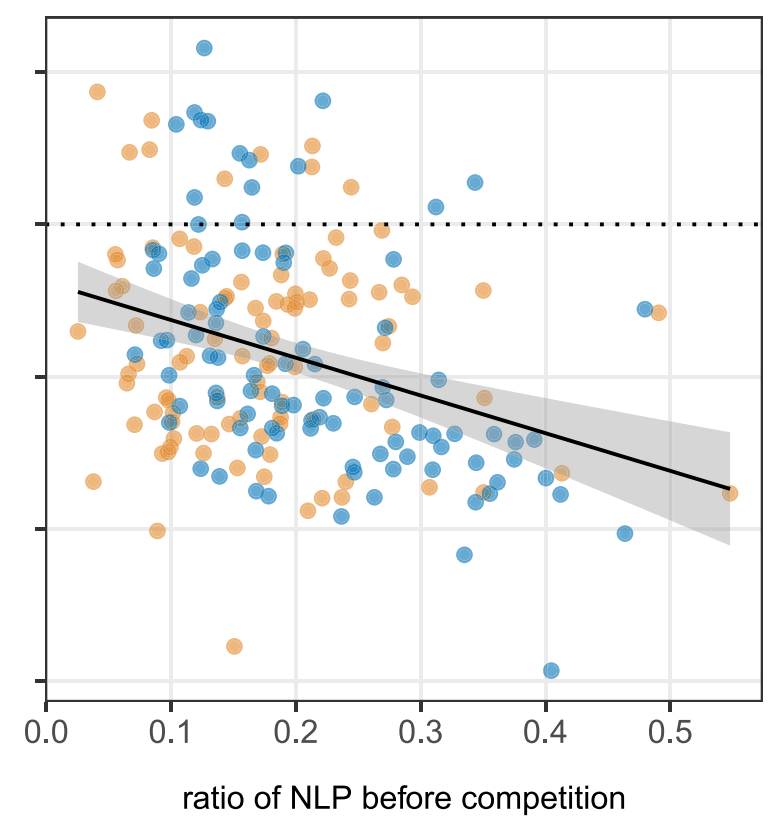

FIGURE 3 Competitive outcomes between pyoverdine producers and NLPs depend on phylogenetic relatedness, but do not differ across the four geographical distance categories. (a) The relative fitness of pyoverdine non- and low-producers (NLPs) increases with the phylogenetic relatedness between producer and NLP but does not differ between the four different distance categories in soil (yellow) and pond (blue). The assay involved 192 isolate combinations (soil: 96, pond: 96), where producers (soil: 69, pond: 66) were competed against NLPs (soil: 6, pond: 6). (b) The relative fitness of NLPs correlates positively with the supernatant effect. (c) The relative fitness of NLPs decreases the more common (i.e. higher in frequency) they were at the beginning of the competition. Solid lines depict significant relationships. Shaded areas are $95 \%$ confidence intervals. Dotted horizontal lines indicate equal fitness of producers and NLPs. The dotted vertical line in (b) indicates no effect of the supernatant on the growth of NLPs

geographical distance nor between habitats (Table 2). Moreover, the relative fitness of the NLPs correlated positively with the supernatant effect $\left(0.792 \pm 0.104, t_{165.1}=7.636, p<.001\right.$; Figure $\left.3 b\right)$. These findings show that phylogenetic relatedness is the main factor determining the outcome of competition for iron between pseudomonads within and across communities. Moreover, they show that the pyoverdine-mediated growth effect in the supernatant assay translates into relative fitness consequences, which highlights the key role of siderophores in determining the outcome of strain interactions.
Finally, the relative fitness of NLPs was also dependent on their initial frequency in the population (Figure 3c). In particular, we found that the relative fitness of NLPs increased the rarer they initially were $\left(-5.930 \pm 1.216, t_{133.1}=-4.878, p<.001\right.$; Figure $\left.3 c\right)$, a pattern predicted by social evolution theory for microbes (RossGillespie et al., 2007). It arises because nonproducers can exploit public goods more efficiently when surrounded by many producers that deliver the exploitable good. We could test for this pattern because, although we adjusted all strains to $\mathrm{OD} 600=0.05$ prior to 
TABLE 2 Statistical analysis of the relative fitness of NLPs in competition with pyoverdine producers. Influence of habitat (soil versus pond), geographical distance, phylogenetic relatedness, the (log-transformed) supernatant effect (as shown in Figure 2) and the starting ratio of the NLPs on their relative fitness in competition with producers

\begin{tabular}{|c|c|c|c|}
\hline & \multicolumn{3}{|c|}{ Relative fitness } \\
\hline & $\chi^{2}$ & $d f$ & $p^{*}$ \\
\hline Habitat & 0.75 & 1 & .388 \\
\hline Geographical distance & 3.16 & 3 & .368 \\
\hline Phylogenetic relatedness & 5.08 & 1 & .024 \\
\hline Supernatant effect & 58.31 & 1 & $<.001$ \\
\hline Starting ratio of NLP & 23.80 & 1 & $<.001$ \\
\hline
\end{tabular}

*Significant $p$-values are given in bold.

the volumetric mixing of 1 NLP: 5 producer units, the actual strain frequency (assessed with flow cytometry) of NLPs varied considerably: 0.025-0.55.

\section{DISCUSSION}

We set out to test whether siderophore-mediated cooperation and cheating can spur patterns of local adaptation in soil and pond communities of Pseudomonas bacteria, sampled across four geographical distance categories. We argued that local adaptation could manifest because pyoverdine, the main siderophore of fluorescent pseudomonads, shows high inter-strain variability in its molecule structure and in the receptor required for siderophore uptake (Butaitè et al., 2017; Ghysels et al., 2004; Meyer et al., 2008; Smith et al., 2005). Consequently, we hypothesized that pyoverdine non- and low-producers (NLPs) might become adapted to efficiently exploit pyoverdines produced by local producers and/or producers might evolve strategies to particularly resist cheating by local nonproducers. While we indeed observed that the level of pyoverdine exploitation and cheating resistance varied considerably between interacting strain pairs, we found little evidence that this variation correlated with geographical distance. Instead, there was a strong signature of phylogenetic relatedness, whereby NLPs were generally better at exploiting the pyoverdines of more closely related producers, irrespective of whether strain pairs originated from the same, close or more distantly related communities. There was one notable exception: within local pond communities the relationship between phylogenetic relatedness and pyoverdine-mediated growth effects was broken. In the sections below, we first discuss possible reasons for the overall weak evidence of local adaptation and then turn to the special interaction pattern observed in local pond communities. Finally, we discuss reasons for why phylogenetic relatedness could be such an important factor driving pyoverdine-mediated social interactions.

There are a number of ecological and evolutionary forces that are known to prevent local adaptation (Blanquart et al., 2013; Kawecki
\& Ebert, 2004; Savolainen et al., 2013). First, high dispersal rates of individuals across the geographical scale sampled could promote gene flow between patches and erode any form of local adaptation. This scenario might apply to the pond habitat, where we found no significant genetic structuring across the geographical scale sampled (Figure 1b). Conversely, significant genetic structuring occurred in soil, suggesting that gene flow is limited in this habitat. Second, local adaptation cannot occur if there is no genetic variation at the trait of interest. This scenario does certainly not apply in our case because our own data show tremendous variation in pyoverdine-mediated growth inhibition and stimulation (Figure 2). Moreover, our previous genetic analysis on the pyoverdine system of a small subset of pond and soil isolates revealed high pyoverdine molecule and receptor diversity, thus providing enough variability for natural selection to act on (Butaitè et al., 2017). Third, local adaptation requires natural selection to be the driving force of evolution. When other factors, such as genetic drift, play a major role, then local adaption cannot occur. While we typically assume that bacterial population sizes are high enough for natural selection to operate, we know too little on the effective size of our Pseudomonas populations to firmly exclude genetic drift as a factor preventing local adaptation. Fourth, frequent temporal variation in environmental conditions can undermine local adaptation. The logic is simple: a beneficial strategy in response to condition A might become unfavourable when conditions change to $B$. It is easy to see how this scenario can apply to fluctuations in abiotic environmental factors. For example, fluctuation in iron availability might affect the selection pressure on NLPs. Under ironrich conditions, NLPs are not dependent on pyoverdine producers, such that any type of NLPs would be selectively favoured, which would undermine local adaptation. In contrast, NLPs depend on producers and a suitable pyoverdine receptor array under iron-limited conditions, which could favour local adaptation. Our previous work revealed that iron availability was consistently low in pond communities, but much higher and more variable in soil communities (Butaitè et al., 2018). Taken together, we reason that increased gene flow in pond and higher environmental fluctuations in soil could be likely explanations for the absence of clear patterns of local adaptation.

We now turn to the local pond communities, where the otherwise prevalent positive relationship between the supernatant effect and the phylogenetic relatedness was absent (Figure 2). This finding recovers our previous result on within-community interaction patterns (Butaite et al., 2017). In this previous paper, we also investigated the genetic basis of pyoverdine-mediated interactions by sequencing the genomes of 24 Pseudomonas isolates. One of the key findings was that most isolates had more than one gene encoding a pyoverdine receptor (median number of receptor homologues per isolate $=4.0$; Table S2). We speculated that (a) horizontal gene transfer could enable isolates to acquire receptor variants of pyoverdine types they do not produce themselves, and (b) frequent horizontal gene transfer would erode the signature of phylogenetic relatedness in ponds at the local scale. We therefore hypothesized that the horizontal acquisition of different pyoverdine receptors happens more often in the diffusive pond environment than in the more structured 
soil environment. In ponds, strain mixing can be high, yet bacteria still adhere to surfaces, conditions that are particularly conducive for horizontal gene transfer (Van Elsas \& Bailey, 2002). To further explore this hypothesis, we re-analysed our receptor genome data from Butaite et al. (2017). We indeed found that pond isolates had significantly higher numbers of pyoverdine receptor homologues than soil isolates (median values 5.5 versus 2.5 ; Wilcoxon rank-sum test: $W=33, p=.0243$; Table S2), whereby NLPs from pond had the highest median number of receptor homologues (median [range] $=8$ [5-10]). These observations are interesting, but what do they mean in the context of local adaptation? Horizontal gene transfer can indeed foster local adaptation (Cordero \& Polz, 2014) such that NLPs could quickly acquire the receptors of the most prevalent local producers. But why were the NLPs then not better at exploiting the local versus the more distant producers? One plausible answer is that the rate of adaptation is similar between NLPs and pyoverdine producers, so that there is simply no overall winner in the antagonistic arms race (Kümmerli et al., 2015), and traces of local adaptation remain thus masked (Kaltz \& Shykoff, 1998).

An important question to address by any study on local adaptation is whether we have looked at the biologically relevant spatial scale. For a Pseudomonas bacterium with a length between 1 to $5 \mu \mathrm{m}$, a geographical scale of $50 \mathrm{~m}$ seems large. For the soil habitat at least, it indeed seems that gene flow is restricted across this scale. However, we also need to examine the lower end of the scale and ask whether isolates from the 'same' community would indeed interact in nature. We do not have a conclusive answer to this question. All we know is that pseudomonads are typically motile (Sampedro et al., 2015), and that pyoverdine secreted on surfaces can be shared between single cells across a range of $100 \mu \mathrm{m}$ (Weigert \& Kümmerli, 2017), a range that can be much larger between macroscopic colonies (Kümmerli et al., 2009). It thus seems reasonable to assume that at least a fraction of strains isolated from our $2 \mathrm{~cm}^{3}$ soil cores might be able to interact with each other in nature. When comparing to other studies, it becomes evident that the scale across which geographical effects are observed varies between bacterial species and habitats, and can cover any range from the centimetre to the kilometre scale (Bruce, West et al., 2017; Hawlena et al., 2010, 2012; Kraemer et al., 2016, 2017; Vogel et al., 2003; Vos \& Velicer, 2008, 2009).

A key finding of our study is that the phylogenetic relatedness between pyoverdine NLPs and pyoverdine producers is the main predictor of (a) the growth effects NLPs experience from the pyoverdine secreted by producers (Figure 2), and (b) the relative fitness of the NLPs in direct competition with producers (Figure 3a). Moreover, we observed that the effects of (a) and (b) correlated positively with each other (Figure $3 \mathrm{~b}$ ), confirming that the pyoverdine-mediated social interactions affect the relative fitness of isolates in direct competition under iron limitation. The strong phylogenetic signature is perhaps surprising at first sight given that isolates have many different pyoverdine receptors (Butaitè et al., 2017), which should allow them to use a range of different pyoverdine types and not only those from close relatives. However, our results now indicate that both absolute and relative fitness consequences seem to be driven by the cognate pyoverdine receptor. In other words, NLPs lost or reduced the ability to produce pyoverdine, but kept their cognate receptor (Butaitè et al., 2017), and they seem to be most efficient in using this receptor to import the (exact or a similar type of) pyoverdine they once produced themselves and is still produced by close relatives. The additional pyoverdine receptors, possibly acquired through horizontal gene transfer, might also be beneficial due to their ability to import noncognate pyoverdines, but their contribution to fitness seems to be smaller and does not override the effect of phylogenetic relatedness in most cases, except within local pond communities. Our interpretations are supported by molecular studies on the laboratory strains $P$. aeruginosa PAO1 (González et al., 2021) and P. protegens Pf-5 (Sexton et al., 2017), which revealed that cognate receptors are preferred over noncognate receptors and that noncognate receptors are conditionally expressed upon sensing of a heterologous pyoverdine. How this sensing exactly works is unclear, but it comes with a time delay (Sexton et al., 2017), probably further reducing the iron-uptake efficiency of noncognate relative to cognate receptors.

Although our main results are based on supernatant assays, involving pyoverdine but also other secreted compounds (e.g. toxins such as phenazines, hydrogen cyanide, etc.), we are confident that the measured fitness effects are indeed predominantly due to pyoverdine (as already demonstrated in Butaite et al., 2017). Our reasoning is based on the fact that all strong supernatant effects (inhibition, stimulation, positive correlation with phylogenetic relatedness) disappeared when the experiment was repeated in iron-rich medium (Figure 2). Note that NLPs received the same supernatantsand hence the same cocktails of secreted compounds-under both iron-limited and iron-rich conditions. The disappearance of the strong supernatant effects under iron-rich conditions hence likely reflects that pyoverdine is overall no longer important for iron scavenging, thereby losing both its stimulatory and inhibitory function on NLPs. Moreover, the experiments in iron-rich medium revealed that the supernatants contain only few (if any) other inhibitory compounds such as toxins, as most supernatants had neutral growth effects on NLPs.

The results from our direct competition assays show that NLPs hardly ever win, despite many of them being able to benefit from the pyoverdines secreted by the producers. There are multiple nonexclusive explanations for this phenomenon. First, the uptake of heterologous pyoverdines by NLPs might be generally less efficient compared to the efficiency by which producers can take up their own pyoverdine. Second, producers might have evolved measures to counteract cheating (Wechsler et al., 2019). For example, we previously found that producers have a higher baseline production of toxins compared to NLPs (Kramer, Lopez Carrasco et al., 2020), which could lower the overall competitiveness of NLPs in direct encounters with producers, especially when toxin production is induced upon sensing the presence of competitors (Lories et al., 2020). Finally, we found that the relative fitness of NLPs increased when being rare in the population (Figure 3c). This simply means that many of the NLPs could become winners in direct competitions when occurring at lower frequency than the ones used in our setup. Negative-frequency-dependent 
fitness effects are common in microbial systems (Diggle et al., 2007; Gilbert et al., 2007; Ross-Gillespie et al., 2007; Zhou et al., 2014) and can occur when secreted compounds are not homogeneously distributed within populations. For example, under static culturing conditions as in our experiments, it is conceivable that NLPs have reduced access to pyoverdine compared to producers, especially when being more common.

In summary, our study contributes to the increasing body of evidence that siderophores are important compounds driving species interactions in a variety of habitats, and in determining the composition and assembly of natural bacterial communities (Bruce, Cooper et al., 2017; Butaitè et al., 2017; Cordero et al., 2012; Gu et al., 2020; Kramer, Özkaya et al., 2020). Although we found little evidence for local adaption with regard to pyoverdine-mediated social interactions, we could show that pyoverdines have strong yet variable effects on bacterial fitness. In particular, we found that (a) the effect of heterologous pyoverdines on non- and lowproducer (NLP) fitness ranges from growth inhibition to promotion; (b) the increased ability to use pyoverdine from other Pseudomonas isolates translates into higher relative fitness in direct competition with producers; and (c) the relative fitness benefit for NLPs increases the more closely they are related to the producers, probably because this increases the likelihood of having a matching receptor based on common ancestry.

\section{ACKNOWLEDGMENTS}

We thank the flow cytometry facility of the University of Zurich and Peter J. Richards for technical support, and Stefan Wyder, Sébastien Wielgoss, Michael Baumgartner and Marta Pinto Carbó for bioinformatical support.

\section{CONFLICT OF INTEREST}

The authors have no conflict of interest to declare.

\section{AUTHOR CONTRIBUTIONS}

$\mathrm{EB}$ and RK designed the experiments; EB conducted the experiments; EB and JK analysed the data; EB, JK and RK interpreted the data and wrote the paper.

\section{PEER REVIEW}

The peer review history for this article is available at https://publo ns.com/publon/10.1111/jeb.13883.

\section{DATA AVAILABILITY STATEMENT}

The data supporting the results in the paper are deposited in the Dryad digital repository (doi: https://doi.org/10.5061/dryad.79cnp 5hvq) and the European Nucleotide Archive under the accession number PRJEB45537 (http://www.ebi.ac.uk/ena)

\section{ORCID}

Elena Butaite (iD https://orcid.org/0000-0003-3573-5130

Jos Kramer (iD https://orcid.org/0000-0003-2155-9295

Rolf Kümmerli iD https://orcid.org/0000-0003-4084-6679

\section{REFERENCES}

Abisado, R. G., Benomar, S., Klaus, J. R., Dandekar, A. A., \& Chandler, J. R. (2018). Bacterial quorum sensing and microbial community interactions. mBio, 9, e02331-e2417.

Abrudan, M. I., Smakman, F., Grimbergen, A. J., Westhoff, S., Miller, E. L., van Wezel, G. P., \& Rozen, D. E. (2015). Socially mediated induction and suppression of antibiosis during bacterial coexistence. Proceedings of the National Academy of Sciences of the United States of America, 112, 11054-11059. https://doi.org/10.1073/pnas.15040 76112

Asfahl, K. L., \& Schuster, M. (2017). Social interactions in bacterial cellcell signaling. FEMS Microbiology Reviews, 41, 92-107. https://doi. org/10.1093/femsre/fuw038

Belotte, D., Curien, J. B., Maclean, R. C., \& Bell, G. (2003). An experimental test of local adaptation in soil bacteria. Evolution, 57, 27-36. https://doi.org/10.1111/j.0014-3820.2003.tb00213.x

Blanquart, F., Kaltz, O., Nuismer, S. L., \& Gandon, S. (2013). A practical guide to measuring local adaptation. Ecology Letters, 16, 1195-1205. https://doi.org/10.1111/ele.12150

Brockhurst, M. A., Harrison, E., Hall, J. P. J., Richards, T., McNally, A., \& MacLean, C. (2019). The ecology and evolution of pangenomes. Current Biology, 29, R1094-R1103. https://doi.org/10.1016/j. cub.2019.08.012

Bruce, J. B., Cooper, G. A., Chabas, H., West, S. A., \& Griffin, A. S. (2017). Cheating and resistance to cheating in natural populations of the bacterium Pseudomonas fluorescens. Evolution, 71, 2484-2495.

Bruce, J. B., West, S. A., \& Griffin, A. S. (2017). Bacteriocins and the assembly of natural Pseudomonas fluorescens populations. Journal of Evolutionary Biology, 30, 352-360.

Butaite, E., Baumgartner, M., Wyder, S., \& Kümmerli, R. (2017). Siderophore cheating and cheating resistance shape competition for iron in soil and freshwater Pseudomonas communities. Nature Communications, 8, 414. https://doi.org/10.1038/s41467-017-00509 $-4$

Butaitè, E., Kramer, J., Wyder, S., \& Kümmerli, R. (2018). Environmental determinants of pyoverdine production, exploitation and competition in natural Pseudomonas communities. Environmental Microbiology, 20, 3629-3642.

Choi, K.-H., \& Schweizer, H. P. (2006). mini-Tn7 insertion in bacteria with single attTn7 sites: Example Pseudomonas aeruginosa. Nature Protocols, 1, 153-161. https://doi.org/10.1038/nprot.2006.24

Cordero, O. X., \& Polz, M. F. (2014). Explaining microbial genomic diversity in light of evolutionary ecology. Nature Reviews Microbiology, 12, 263-273. https://doi.org/10.1038/nrmicro3218

Cordero, O. X., Ventouras, L.-A., DeLong, E. F., \& Polz, M. F. (2012). Public good dynamics drive evolution of iron acquisition strategies in natural bacterioplankton populations. Proceedings of the National Academy of Sciences of the United States of America, 109, 20059-20064. https:// doi.org/10.1073/pnas.1213344109

Diggle, S. P., Griffin, A. S., Campell, G. S., \& West, S. A. (2007). Cooperation and conflict in quorum-sensing bacterial populations. Nature, 450 , 411-414. https://doi.org/10.1038/nature06279

Faraldo-Gómez, J. D., \& Sansom, M. S. P. (2003). Acquisition of siderophores in gram-negative bacteria. Nature Reviews Molecular Cell Biology, 4, 105-116. https://doi.org/10.1038/nrm1015

Ghoul, M., \& Mitri, S. (2016). The ecology and evolution of microbial competition. Trends in Microbiology, 24, 833-845. https://doi. org/10.1016/j.tim.2016.06.011

Ghoul, M., West, S. A., Diggle, S. P., \& Griffin, A. S. (2014). An experimental test of whether cheating is context dependent. Journal of Evolutionary Biology, 27, 551-556. https://doi.org/10.1111/jeb.12319

Ghyselinck, J., Coorevits, A., Van Landschoot, A., Samyn, E., Heylen, K., $\&$ De Vos, P. (2013). An rpoD gene sequence based evaluation of cultured Pseudomonas diversity on different growth media. Microbiology, 159, 2097-2108. https://doi.org/10.1099/mic.0.068031-0 
Ghysels, B., Thi Min Dieu, B., Beatson, S. A., Pirnay, J.-P., Ochsner, U. A., Vasil, M. L., \& Cornelis, P. (2004). FpvB, an alternative type I ferripyoverdine receptor of Pseudomonas aeruginosa. Microbiology, 150, 1671-1680.

Gilbert, O. M., Foster, K. R., Mehdiabadi, N. J., Strassmann, J. E., \& Queller, D. C. (2007). High relatedness maintains multicellular cooperation in a social amoeba by controlling cheater mutants. Proceedings of the National Academy of Sciences of the United States of America, 104, 8913-8917. https://doi.org/10.1073/pnas.0702723104

Giraud, T., Koskella, B., \& Laine, A. L. (2017). Introduction: Microbial local adaptation: Insights from natural populations, genomics and experimental evolution. Molecular Ecology, 26, 1703-1710.

González, J., Salvador, M., Ozkaya, O., Spick, M., Reid, K., Costa, C., Bailey, M. J., Avignone Rossa, C., Kümmerli, R., \& Jimenez, J. I. (2021). Loss of a pyoverdine secondary receptor in Pseudomonas aeruginosa results in a fitter strain suitable for population invasion. ISME Journal, $15,1330-1343$.

Gould, W. D., Hagedorn, C., Bardinelli, T. R., \& Zablotowicz, R. M. (1985). New selective media for enumeration and recovery of fluorescent pseudomonads from various habitats. Applied and Environment Microbiology, 49, 28-32. https://doi.org/10.1128/ aem.49.1.28-32.1985

Greischar, M. A., \& Koskella, B. (2007). A synthesis of experimental work on parasite local adaptation. Ecology Letters, 10, 418-434. https:// doi.org/10.1111/j.1461-0248.2007.01028.x

Gu, S., Wei, Z., Shao, Z., Friman, V. P., Cao, K., Yang, T., Kramer, J., Wang, X., Li, M., Mei, X., Xu, Y., Shen, Q., Kümmerli, R., \& Jousset, A. (2020). Competition for iron drives phytopathogen control by natural rhizosphere microbiomes. Nature Microbiology, 5, 1002-1010. https://doi. org/10.1038/s41564-020-0719-8

Hawlena, H., Bashey, F., \& Lively, C. M. (2010). The evolution of spite: Population structure and bacteriocin-mediated antagonism in two natural populations of Xenorhabdus bacteria. Evolution, 64, 31983204. https://doi.org/10.1111/j.1558-5646.2010.01070.x

Hawlena, H., Bashey, F., \& Lively, C. M. (2012). Bacteriocin-mediated interactions within and between coexisting species. Ecology and Evolution, 2, 2516-2521. https://doi.org/10.1002/ece3.354

Hereford, J. (2009). A quantitative survey of local adaptation and fitness trade-offs. American Naturalist, 173, 579-588. https://doi. org/10.1086/597611

Hibbing, M. E., Fuqua, C., Parsek, M. R., \& Peterson, S. B. (2010). Bacterial competition: Surviving and thriving in the microbial jungle. Nature Reviews Microbiology, 8, 15-22. https://doi.org/10.1038/ nrmicro2259

Hider, R. C., \& Kong, X. (2010). Chemistry and biology of siderophores. Natural Products Reports, 27, 637-657. https://doi.org/10.1039/ b906679a

Kaltz, O., \& Shykoff, J. A. (1998). Local adaptation in host-parasite systems. Heredity, 81, 361-370. https://doi.org/10.1046/j.1365-2540. 1998.00435.x

Kawecki, T. J., \& Ebert, D. (2004). Conceptual issues in local adaptation. Ecology Letters, 7, 1225-1241. https://doi.org/10.1111/j.1461-0248. 2004.00684.x

Kinkel, L. L., Schlatter, D. C., Xiao, K., \& Baines, A. D. (2014). Sympatric inhibition and niche differentiation suggest alternative coevolutionary trajectories among Streptomycetes. ISME Journal, 8, 249-256. https:// doi.org/10.1038/ismej.2013.175

Kraemer, S. A., \& Boynton, P. J. (2017). Evidence for microbial local adaptation in nature. Molecular Ecology, 26, 1860-1876.

Kraemer, S. A., \& Kassen, R. (2015). Patterns of local adaptation in space and time among soil bacteria. American Naturalist, 185, 317-331. https://doi.org/10.1086/679585

Kraemer, S. A., \& Kassen, R. (2016). Temporal patterns of local adaptation in soil pseudomonads. Proceedings of the Royal Society B: Biological Sciences, 283, 20161652.
Kraemer, S. A., Soucy, J. P. R., \& Kassen, R. (2017). Antagonistic interactions of soil pseudomonads are structured in time. FEMS Microbiology Ecology, 93, 1-9. https://doi.org/10.1093/femsec/fix046

Kraemer, S. A., Wielgoss, S., Fiegna, F., \& Velicer, G. J. (2016). The biogeography of kin discrimination across microbial neighbourhoods. Molecular Ecology, 25, 4875-4888. https://doi.org/10.1111/ mec.13803

Kramer, J., Lopez Carrasco, M. A., \& Kümmerli, R. (2020). Positive linkage between bacterial social traits reveals that homogeneous rather than specialised behavioral repertoires prevail in natural Pseudomonas communities. FEMS Microbiology Ecology, 96, fiz185. https://doi. org/10.1093/femsec/fiz185

Kramer, J., Özkaya, Ö., \& Kümmerli, R. (2020). Bacterial siderophores in community and host interactions. Nature Reviews Microbiology, 18, 152-163. https://doi.org/10.1038/s41579-019-0284-4

Kümmerli, R., Griffin, A. S., West, S. A., Buckling, A., \& Harrison, F. (2009). Viscous medium promotes cooperation in the pathogenic bacterium Pseudomonas aeruginosa. Proceedings of the Royal Society B, 276, 3531-3538.

Kümmerli, R., Santorelli, L., Granato, E., Dumas, Z., Dobay, A., Griffin, A. S., \& West, S. A. (2015). Co-evolutionary dynamics between public good producers and cheats in the bacterium Pseudomonas aeruginosa. Journal of Evolutionary Biology, 28, 2264-2274.

Kümmerli, R., Schiessl, K. T., Waldvogel, T., McNeill, K., \& Ackermann, M. (2014). Habitat structure and the evolution of diffusible siderophores in bacteria. Ecology Letters, 17, 1536-1544. https://doi.org/10.1111/ ele.12371

Lee, W., van Baalen, M., \& Jansen, V. A. A. (2012). An evolutionary mechanism for diversity in siderophore producing bacteria. Ecology Letters, 15, 119-125. https://doi.org/10.1111/j.1461-0248.2011.01717.x

Leimu, R., \& Fischer, M. (2008). A meta-analysis of local adaptation in plants. PLoS One, 3, e4010. https://doi.org/10.1371/journ al.pone.0004010

Lories, B., Roberfroid, S., Dieltjens, L., De Coster, D., Foster, K. R., \& Steenackers, H. P. (2020). Biofilm bacteria use stress responses to detect and respond to competitors. Current Biology, 30, 1231-1244. https://doi.org/10.1016/j.cub.2020.01.065

Madeira, F., Park, Y. M., Lee, J., Buso, N., Gur, T., Madhusoodanan, N., Basutkar, P., Tivey, A. R. N., Potter, S. C., Finn, R. D., \& Lopez, R. (2019). The EMBL-EBI search and sequence analysis tools APIs in 2019. Nucleic Acids Research, 47, W636-W641. https://doi. org/10.1093/nar/gkz268

Meyer, J. M., Gruffaz, C., Raharinosy, V., Bezverbnaya, I., Schäfer, M., \& Budzikiewicz, H. (2008). Siderotyping of fluorescent Pseudomonas: Molecular mass determination by mass spectrometry as a powerful pyoverdine siderotyping method. BioMetals, 21, 259-271. https:// doi.org/10.1007/s10534-007-9115-6

Mulet, M., Bennasar, A., Lalucat, J., \& García-Valdés, E. (2009). An rpoDbased PCR procedure for the identification of Pseudomonas species and for their detection in environmental samples. Molecular and Cellular Probes, 23, 140-147. https://doi.org/10.1016/j. mcp.2009.02.001

Nogueira, T., Rankin, D. J., Touchon, M., Taddei, F., Brown, S. P., \& Rocha, E. P. C. (2009). Horizontal gene transfer of the secretome drives the evolution of bacterial cooperation and virulence. Current Biology, 19, 1683-1691. https://doi.org/10.1016/j.cub.2009.08.056

Ross-Gillespie, A., Gardner, A., West, S. A., \& Griffin, A. S. (2007). Frequency dependence and cooperation: Theory and a test with bacteria. American Naturalist, 170, 331-342. https://doi. org/10.1086/519860

Sampedro, I., Parales, R. E., Krell, T., \& Hill, J. E. (2015). Pseudomonas chemotaxis. FEMS Microbiology Reviews, 39, 17-46.

Savolainen, O., Lascoux, M., \& Merila, J. (2013). Ecological genomics of local adaptation. Nature Reviews Genetics, 14, 807-820. https://doi. org/10.1038/nrg3522 
Schalk, I. J., \& Guillon, L. (2013). Pyoverdine biosynthesis and secretion in Pseudomonas aeruginosa: Implications for metal homeostasis. Environmental Microbiology, 15, 1661-1673.

Sexton, D. J., Glover, R. C., Loper, J. E., \& Schuster, M. (2017). Pseudomonas protegens Pf- 5 favours self-produced siderophore over free-loading in interspecies competition for iron. Environmental Microbiology, 19, 3514-3525.

Silby, M. W., Winstanley, C., Godfrey, S. A. C., Levy, S. B., \& Jackson, R. W. (2011). Pseudomonas genomes: Diverse and adaptable. FEMS Microbiology Reviews, 35, 652-680.

Smith, E. E., Sims, E. H., Spencer, D. H., Kaul, R., \& Olson, M. V. (2005). Evidence for diversifying selection at the pyoverdine locus of Pseudomonas aeruginosa. Journal of Bacteriology, 187, 2138-2147.

Van Elsas, J. D., \& Bailey, M. J. (2002). The ecology of transfer of mobile genetic elements. FEMS Microbiology Ecology, 42, 187-197. https:// doi.org/10.1111/j.1574-6941.2002.tb01008.x

Visca, P., Imperi, F., \& Lamont, I. L. (2007). Pyoverdine siderophores: From biogenesis to biosignificance. Trends in Microbiology, 15, 22-30. https://doi.org/10.1016/j.tim.2006.11.004

Vogel, J., Normand, P., Thioulouse, J., Nesme, X., \& Grundmann, G. L. (2003). Relationship between spatial and genetic distance in Agrobacterium spp. in 1 cubic centimeter of soil. Applied and Environment Microbiology, 69, 1482-1487.

Vos, M., \& Velicer, G. J. (2008). Isolation by distance in the spore-forming soil bacterium Myxococcus xanthus. Current Biology, 18, 386-391. https://doi.org/10.1016/j.cub.2008.02.050

Vos, M., \& Velicer, G. J. (2009). Social conflict in centimeter and globalscale populations of the bacterium Myxococcus xanthus. Current Biology, 19, 1763-1767. https://doi.org/10.1016/j.cub.2009.08.061

Wechsler, T., Kümmerli, R., \& Dobay, A. (2019). Understanding policing as a mechanism of cheater control in cooperating bacteria. Journal of Evolutionary Biology, 32, 412-424. https://doi.org/10.1111/jeb.13423
Weigert, M., \& Kümmerli, R. (2017). The physical boundaries of public goods cooperation between surface-attached bacterial cells. Proceedings of the Royal Society B, 284, 20170631. https://doi. org/10.1098/rspb.2017.0631

West, S. A., Diggle, S. P., Buckling, A., Gardner, A., \& Griffin, A. S. (2007). The social lives of microbes. Annual Review of Ecology Evolution and Systematics, 38, 53-77. https://doi.org/10.1146/annurev.ecols ys.38.091206.095740

Zhou, L., Slamti, L., Nielsen-LeRoux, C., Lereclus, D., \& Raymond, B. (2014). The social biology of quorum sensing in a naturalistic host pathogen system. Current Biology, 24, 2417-2422. https://doi. org/10.1016/j.cub.2014.08.049

\section{SUPPORTING INFORMATION}

Additional supporting information may be found online in the Supporting Information section.

How to cite this article: Butaitè, E., Kramer, J., \& Kümmerli, R. (2021). Local adaptation, geographical distance and phylogenetic relatedness: Assessing the drivers of siderophore-mediated social interactions in natural bacterial communities. Journal of Evolutionary Biology, 34, 1266-1278. https://doi.org/10.1111/jeb.13883 